obtained from healthy controls by centrifugation over Polymorphoprep $^{\mathrm{TM}}$, was quantified in Boyden chambers.

Results No significant difference in the relative killing of E.coli K12 (-14 $\pm 11 \%$ vs. $-45 \pm 9 \%)$ and Staph. aureus $(-52 \pm 4 \%$ vs. $-63 \pm 5 \%)$ nor in the relative survival of AIEC HM605 $(+50 \pm 26 \%$ vs. $+8 \pm 22 \%)$ within monocyte-derived macrophages was seen (healthy controls vs. Crohn's disease respectively; $\mathrm{n}=10$ each group, ANOVA). TNF $\alpha$, IL-6 and IL-8 production were not significantly different between the two groups and macrophage mediated neutrophil chemotaxis was equivalent. Smoking status did not affect bacterial survival, with no differences observed in killing between current smokers, exsmokers and non-smokers.

Conclusion AIEC are ineffectively killed by both Crohn's disease and healthy macrophages. Macrophages from patients with Crohn's disease do not appear to have an inherent defect in killing and exhibit equivalent ability to induce neutrophil chemotaxis relative to controls. These data suggest circulating inhibitors of Neutrophil chemotaxis may explain the previously observed defective neutrophil chemotaxis and bacterial clearance in vivo.

Disclosure of Interest P. Flanagan Grant/research support from: Awarded a Shire innovation fund for SpRs, S. Subramanian: None Declared, B. Campbell: None Declared, J. Rhodes Consultant for: A member of advisory boards for Atlantic, Procter and Gamble and Falk, Speaker bureau with: Has received speaking honoraria from Abbott, Falk, Ferring, Glaxo Smith Kline, Procter and Gamble, Schering Plough, Shire and Wyeth, Conflict with: With the University of Liverpool and Provexis UK, holds a patent for use of a soluble fibre preparation as maintenance therapy for Crohn's disease plus a patent pending for its use in antibiotic-associated diarrhoea.

\section{PWE-092 SYMPTOM RESPONSE FOLLOWING ADVICE ON A DIET LOW IN SHORT-CHAIN FERMENTABLE CARBOHYDRATES (FODMAPS) FOR FUNCTIONAL BOWEL SYMPTOMS IN PATIENTS WITH IBD}

${ }^{1} \mathrm{~T}$ Joyce, ${ }^{1,2} \mathrm{H}$ Staudacher, ${ }^{2} \mathrm{~K}$ Whelan, ${ }^{2,3} \mathrm{P}$ Irving ${ }^{*},{ }^{1,2,3} \mathrm{M}$ Lomer. ${ }^{1}$ Dietetics, Guy's and St Thomas' NHS Foundation Trust, London, UK; ${ }^{2}$ Diabetes and Nutritional Sciences, King's College London, London, UK; ${ }^{3}$ Gastroenterology, Guy's and St Thomas' NHS Foundation Trust, London, UK

\subsection{6/gutjnl-2014-307263.352}

Introduction The low FODMAP diet is an effective dietary intervention for people with functional bowel disorders (FBD), which are common in patients with inflammatory bowel disease (IBD). One study has reported that half of patients with IBD report improvements in abdominal pain, bloating, flatulence and diarrhoea after following a low FODMAP diet. Up to $70 \%$ of patients reported adherence to the diet. We aimed to assess the effectiveness of low FODMAP diet advice in patients with IBD in the UK.

Methods Patients with inactive IBD and FBD (as diagnosed by their gastroenterologist), who had been referred to the dietitian and advised on a low FODMAP diet were included in this evaluation of clinical practice. Symptoms were measured pre and post (at least 6 weeks) low FODMAP dietary advice as part of normal clinical practice using the global symptom question (GSQ)'Do you currently have satisfactory relief of your gut symptoms?' and the gastrointestinal symptom rating scale (GSRS). Stool frequency was considered 'normal' if between 2-3 times per week to 2-3 times per day. Types 3-5 on the Bristol stool form scale were considered normal stool consistency. Comparisons were made using the $\mathrm{McNemar}$ test for categorical data and a paired t-test for continuous data.

Results Data from 35 patients with IBD (17 ulcerative colitis, 17 Crohn's disease, one IBD unclassified, $63 \%$ female, mean age $39 y)$ were analysed. There was a significant increase in the number of patients reporting positively to the GSQ (n[\%] pre: 8 [23] versus post: 29 [83]; $\mathrm{p}<0.001)$ and the GSRS composite score (mean [SD] pre: 1.25 [0.48] versus post: 0.77 [0.45]; p < 0.001). Fewer patients reported symptoms including abdominal pain, bloating, flatulence and urgency, increased stool frequency, and type 6 or 7 Bristol stool form post dietary advice compared with pre dietary advice (Table).

Conclusion The low FODMAP diet appears to be an effective treatment option for patients with IBD and FGD particularly for those with symptoms of abdominal bloating, flatulence, faecal urgency and lethargy. However, this is an evaluation of clinical practice and prospective randomised controlled evidence in IBD is currently lacking.

Disclosure of Interest None Declared.

\section{PWE-093 SPLITTING THE NORMAL DAILY DOSE OF THIOGUANINE MAY BE EFFICACIOUS TREATMENT FOR INFLAMMATORY BOWEL DISEASE AND AVOID HEPATIC TOXICITY}

${ }^{1} \mathrm{P}$ Pavlidis*, ${ }^{1} \mathrm{~A}$ Ansari, ${ }^{2} \mathrm{D}$ Duley, ${ }^{3} \mathrm{I}$ Oancea, ${ }^{4} \mathrm{~T}$ Florin. ${ }^{1}$ Digestive Diseases, Surrey and Sussex Healthcare NHS Trust, East Surrey Hospital, Redhill, UK; ${ }^{2}$ Pharmacy, University of Queensland, Woolloongabba, Australia; ${ }^{3}$ Translational Research Institute, Immunity, Infection, and Inflammation Program, Mater Research Institute, University of Queensland, Woolloongabba, Australia; ${ }^{4}$ Mater Research Institute, University of Queensland, South Brisbane, Australia

\subsection{6/gutjpl-2014-307263.353}

Introduction 6-thioguanine (TG) is a treatment for inflammatory bowel disease (IBD). However, its association with nodular regenerative hyperplasia (NRH) and portal hypertension has

\begin{tabular}{|c|c|c|c|c|c|c|c|}
\hline & Pre & Post & & & Pre & Post & \\
\hline Symptom & n (\%) & n (\%) & $\mathrm{p}$ & Symptom & n (\%) & n (\%) & $\mathrm{p}$ \\
\hline Abdominal pain & $13(37)$ & $6(17)$ & 0.039 & Nausea & $3(9)$ & $0(0)$ & 0.250 \\
\hline Abdominal bloating & $25(71)$ & $9(26)$ & $<0.001$ & Heartburn & $4(11)$ & $3(9)$ & 1.000 \\
\hline Flatulence & $24(69)$ & $7(20)$ & $<0.001$ & Acid regurgitation & $4(11)$ & $1(3)$ & 0.250 \\
\hline Belching & $6(17)$ & $3(9)$ & 0.508 & Lethargy & 27 (77) & $14(40)$ & 0.002 \\
\hline Borborygmi & $17(49)$ & $6(17)$ & 0.003 & Stool frequency $>3$ times/day & $17(49)$ & $8(23)$ & 0.012 \\
\hline Faecal urgency & $22(63)$ & $9(26)$ & 0.002 & Type 6 or 7 Bristol stool form & $12(34)$ & $9(26)$ & 0.549 \\
\hline Incomplete evacuation & $9(26)$ & $8(23)$ & 1.000 & & & & \\
\hline
\end{tabular}


restricted its utilisation. A murine model has provided evidence that splitting a normal daily dose of TG could prevent exposure of the liver to harmful levels of TG; furthermore safety has not been an issue in human studies with low dose TG.

Methods We report from two centres on a retrospective experience of the safety and efficacy of an oral split-daily TG dose regimen, to avoid any individual dose $>0.3 \mathrm{mg} / \mathrm{kg}$, in $62 \mathrm{IBD}$ patients who were unresponsive or had suffered adverse drug reactions to conventional therapies including thiopurines (60), biologics (18) and calcineurin inhibitors (17). Clinical response was measured using the Harvey Bradshaw Index for Crohn's, or the Simple Clinical Colitis Score for ulcerative and indeterminate colitis. Patients were followed regularly in clinic with bloods, liver biopsy (9) and progress ultrasound at 6 months (21) or MR imaging (2).

Results Median duration of TG treatment was 7.8 (0.3-45) months. Median TG dose used was $0.6(0.3-1) \mathrm{mg} / \mathrm{kg} / \mathrm{d}$. Of patients attaining 6 months of TG therapy, 91\% (19/21) of Crohn's patients and 71\% (27/38) with ulcerative or indeterminate colitis had a clinically significant response, off steroids. At study end, 33 (53\%) patients maintained their good clinical response off steroids; 12 of these had continued with concomitant biologic or calcineurin inhibitor therapy.

Previous thiopurine-related adverse reactions were not encountered. $29(47 \%)$ patients withdrew from the study because of loss to follow-up (5), medical adverse events (2) or surgery (22). Possible early NRH was found on liver biopsy in 1 patient who was heterozygote-deficient for thiopurine methyltransferase (TPMT); the patient continued TG at a lower dose. TG was discontinued in a patient found to have NRH and concomitant anti-phospholipid syndrome. There was one successful term pregnancy; cord blood and breast milk TG were low.

Conclusion A split-dose regimen of TG appeared well-tolerated, efficacious and safe for selected IBD patients. Close monitoring, knowledge of TPMT and exclusion of risk factors for NRH prior to treatment are warranted to maximise safety.

\section{REFERENCES}

Dubinsky MC, Vasiliauskas EA, Singh $H$, et al. 6-thioguanine can cause serious liver injury in inflammatory bowel disease patients. Gastroenterology 2003:125:298-303 Oancea I, Png CW, Das I, et al. A novel mouse model of veno-occlusive disease provides strategies to prevent thioguanine-induced hepatic toxicity. Gut 2013:62:594605

Disclosure of Interest None Declared.

\section{PWE-094 SETTING STANDARDS BY DEFINING THE AIMS AND OPTIMAL DESIGN OF THE INFLAMMATORY BOWEL DISEASE (IBD) MULTIDISCIPLINARY TEAM (MDT) MEETING}

${ }^{1,2}{ }^{2}$ Morar*,${ }^{3}$ J Read, ${ }^{2} \mathrm{~S}$ Arora, ${ }^{1,2} \mathrm{~A}$ Hart, ${ }^{2} \mathrm{~N}$ Sevdalis, ${ }^{1,2} \mathrm{O}$ Faiz, ${ }^{3} \mathrm{C}$ Edwards. ${ }^{1}$ St Mark's Hospital; ${ }^{2}$ Imperial College, London, UK; ${ }^{3}$ South Devonshire NHS Foundation Trust, Torbay, UK

10.1136/gutjnl-2014-307263.354

Introduction The National IBD Audit revealed $75 \%$ of participating institutions undertake a weekly MDT meeting for IBD patients. There is however little evidence of its efficacy in this context and currently there is no guidance on how this intervention may be standardised and used effectively. ${ }^{1-3}$ Providing a standardised framework for the IBD MDT meeting will enhance its capacity to establish effective quality improvement. The aim of this study is to use national expert consensus to define the aims, optimal design, format and function of an IBD MDT meeting.
Methods 25 semistructured interviews were undertaken with a multidisciplinary sample (5 surgeons, 5 gastroenterologists, 5 IBD nurse specialists, 5 pathologists and 5 radiologists), from 2 UK regions: the Southwest of England and London. Interviews were audiotaped and transcribed verbatim. A standardised interview protocol with a clearly defined coding framework was used. The interview protocol explored key themes encompassing the optimal design format of the IBD MDT:

1. Purpose

2. Processes

3. Logistics

4. Redesign

Results 28 interviews were performed across a multidisciplinary sample of healthcare professionals. Thematic analysis and coding demonstrated common markers for each theme. High ranking markers for each theme included:

1. Purpose: Requires multi-disciplinary input; to share collective expertise; and to improve patient outcome.

2. Processes: Good attendance; sharing workload with colleagues; proactive discussions; core members being clinicians, surgeons, radiologists, pathologists and nurse specialists all with IBD interests; facilities required including IT and an appropriate space to meet; provisions for internal feedback to the IBD MDT on MDT decision outcomes; submitting names in advance; an MDT coordinator.

3. Logistics: Duration of $1 \mathrm{~h}$; once a week; protected time; selective cases.

4. Redesign: Single centre each running their own IBD MDT; 'hub and spoke' model.

Conclusion Defining key elements for an optimal design format for the IBD MDT is necessary to ensure quality of care and reduce variation in care standards. This study demonstrates the methodology used for construction of provisional standards for the IBD MDT through interviews from a multidisciplinary group. Selection and adjustments of these standards through expert consensus are required to validate measures.

\section{REFERENCES}

1 UK IBD Steering Group 2007 IBD Audit 2006: National Results for the Organisation and Process of IBD Care in the UK

2 Group 2009 IBD Audit 2008: National Results for the Organisation and Process of IBD Care in the UK

3 IBD Standards Working Group 2009 Quality Care: Service Standards for the Healthcare of People who have Inflammatory Bowel Disease (IBD)

Disclosure of Interest None Declared.

\section{PWE-095 FAECAL CALPROTECTIN IS USEFUL IN PREDICTING LONG TERM DISEASE RECURRENCE IN POST-OPERATIVE CROHN'S}

R Perowne*, C Lamb, RA Speight, M Price, J Mansfield. Royal Victoria Infirmary, NE3 4LT, UK

\subsection{6/gutjnl-2014-307263.355}

Introduction The concept of using faecal biomarkers to predict prognosis and direct treatment in patients with Crohn's disease is attractive but long term follow-up data is lacking.

A cohort of 51 patients with previous ileal resections for Crohn's disease provided a one-off stool sample for faecal calprotectin (FC). These patients were followed up for 5 years to assess whether FC could predict disease progression in the long term.

Methods Patients were identified from a database of patients who had participated in a previous study evaluating the use of 